\title{
LOGIC AND INTENSIONALITY
}

\author{
GUIDO IMAGUIRE
}

Universidade Federal do Rio de Janeiro

\begin{abstract}
There are different ways we use the expressions "extension" and "intension". I specify in the first part of this paper two basic senses of this distinction, and try to show that the old metaphysical sense, by means of particular instance vs. universal, is more fundamental than the contemporary sense by means of substitutivity. In the second part, I argue that logic in general is essentially intensional, not only because logic is a rule-guided activity, but because even the extensional definition of a logic system presupposes an intensional notion of logical consequence.
\end{abstract}

Keywords: Extension, intension, logical consequence.

The most distinctive feature of a great thinker is his capacity to show that some of our most established convictions are not so well founded as we think. Quine's criticisms on the notions of meaning and analyticity, Wittgenstein's criticisms on the traditional essentialistic notion of concept, Kripke's criticisms on our intuitive identification of necessity and apriority are some examples of this kind of challenging insights of big thinkers. Newton da Costa has the merit of showing that ex falso sequitur quodlibet ( $\alpha \wedge \neg \alpha \vdash \beta$ ) is not, as we accepted for many centuries, a necessary principle for every logic. Many logicians tried to construct in the last century new logics suspending one or another principle: suspend monotonicity and you get non-monotonic logic, suspend bivalence and you get many-valued logic, etc. The enormous plurality of systems of logic leads many to give up the idea that there is a common core of all kinds of logic. In this paper I shall discuss the question of whether there is something that can be considered an essential property of every system of logic. Indeed, I will argue that intensionality-in a particular meaning of this ambiguous word-is an essential feature of every kind of logic.

Of course, there are extensional and intensional formal systems of logic. In general we consider a system of logic intensional when its variables range over a domain which includes intensional entities like possible objects, propositions, concepts, qualities, or when some extensional principle is not valid for it. But, provided the characterization of extensionality and intensionality I will give in the first section of this paper, there is a specific (and more basic) sense of "intensionality", according to which we can say that every system of logic, and not only the so called "intensional logics", has an intensional nature.

Principia 14(1): 111-24 (2010).

Published by NEL — Epistemology and Logic Research Group, Federal University of Santa Catarina (UFSC), Brazil. 


\section{Two Views on Intensionality and Extensionality}

There are two basic views concerning the extension-intension distinction. The first view is more philosophical, we could even say "metaphysical", and goes back to Plato. His theory of pure forms was intended as a solution to the problem of "unity over plurality". What is the identical nature e.g. of the different instances of red? What make different red objects red? The theory of universals as unlocated (or located in a "heaven") pure forms is his solution: every red thing participates in (instantiates) the pure form of redness. In this sense redness qua universal is a kind of rule that determines the extension of red things. But there is a well known problem here. Let us recall the old example: man and featherless biped are concepts with different intensions, but both correspond to (determine) the same extension (in the actual world).

In this first view, the extension is a collection of particular instances, and the intension is a kind of corresponding rule for determining this collection. To have an intension corresponding to a given extension is always something desirable in practice and in theory. In computer science, the rule or intension allows a kind of "compression of information": instead of an extensive list of data, we can give a simple rule for generating this complete list. But also in metaphysics is the intension desirable: when you discover the intension that precisely corresponds to the collection of all particular objects that fall under a concept (e.g. each particular object truly called "human") you probably (not necessarily) get the "essence" of this concept (humanity).

If there is a corresponding intension (a defining concept) to every extension, or a corresponding extension to every intension, is one of the most difficult questions of philosophy. Many sceptical arguments in modern and contemporary philosophy are based on the insight that an extension-conceived as a set or particular instancescannot determine (but only sub-determine) a corresponding intension. All these arguments can be schematically represented by the following graphic:

$$
R \quad \leftrightarrows \quad i_{1}, i_{2}, i_{3}, \ldots
$$

where $R$ represents the rule (in my sense "intension") that corresponds to the collection of particular instances $i_{1}, i_{2}, i_{3}, \ldots$ (the "extension"). The double arrow represents the mysterious relation between them, and different conceptions will emerge according to the direction you privilege. When you start from the extensions on the right side and "create" the rule $\mathrm{R}$ on the left, you probably have nominalistic tendencies; when you start from the rule $R$ that defines or determines the collection of particulars instances of the right, you possibly sympathise with Platonism. I think this can be applied to many different areas and problems of philosophy. But this is not our issue in this paper.

Principia 14(10): 111-24 (2010). 
The sceptical arguments I meant in the last paragraph are the following. The set of all observed white swans $\left(i_{1}, i_{2}, i_{3}, \ldots\right)$ does not determine (support or verify in definitive manner) the law $(R)$ that all swans are white, the set of all observed green (or grue) smaragds $\left(i_{1}, i_{2}, i_{3}, \ldots\right)$ does not determine the law $(R)$ that all smaragds are green, the set of all utterances of "gavagai" $\left(i_{1}, i_{2}, i_{3}, \ldots\right)$ does not determine the meaning $(R)$ of this word, neither does the corresponding reference (or ontology), the set $\{0,1,2,3, \ldots\}\left(i_{1}, i_{2}, i_{3}, \ldots\right)$ determine the correct rule that defines the rule of generation of natural numbers $(R)$, etc. There is a gap between extensions and intensions that nobody can bypass. In Wittgenstein's words: a rule or function is not another way to give the same that a list gives. In a mathematical example:

$$
f(x): x^{2}
$$

is not the same as

$$
\{\langle 0,0\rangle,\langle 1,1\rangle,\langle 2,4\rangle,\langle 3,9\rangle, \ldots\},
$$

even if we could substitute the dots by the complete list. The acceptance of arbitrary functions (set of ordered pairs without any corresponding rule) by Lejeune-Dirichlet was one of the most controversial revolutions in the history of mathematics. Classical theory of concepts is another case of this extension-intension priority debate: what is at stake here is the question if all particular instances of application of a given concept, like game, has a common feature (if there is a rule) or not.

In "Extensionality" R. B. Marcus (1971) offers a good analysis of extensionality in the sense I call here "the second view". According to this view, extensionality can be defined in terms of the principle (or better: one of the many principles) of substitutivity.

Propositional logic is said to be extensional because its connectives are extensional functions: the truth value of a complex sentence is completely determined by the truth values of its component sentences, i.e. each sentence can be replaced by another sentence of the same truth value by preservation of the truth value of the complex sentence, independently on the meaning and on the modal status of the substituted sentences. Predicate logic is said to be extensional, (1) because of the extensionality of propositional connectives just explained, and (2) because any two predicates $F$ and $G$, which determine exactly the same extension $(\forall x(F x \leftrightarrow G x))$ can substitute each other in every formulae salva veritate. I.e. only the extension and not the intension of a predicate is relevant for determining the true value of a formula. Something analogous can be said about set theory, mereology, extensional contexts in semantics, etc. We can say in general that a context is extensional when different particular instances of a given equivalent class are considered in this context identical and inter-substitutable for some functional purposes. Or, alternatively, we can say that a context is extensional when some (intensional) aspect is 
deliberately ignored. This can be represented by a simplified schema:

$$
\left.\begin{array}{l}
\mathbf{E}_{i}=\left\{i_{1}, i_{2}, i_{3}, \ldots\right\} \\
\mathbf{E}_{j}=\left\{j_{1}, j_{2}, j_{3}, \ldots\right\} \\
\cdots
\end{array}\right\} \quad f(\ldots, \ldots) \Rightarrow\left\{\begin{array}{c}
A \\
B \\
\ldots
\end{array}\right.
$$

The equivalent classes $\mathbf{E}_{i}, \mathbf{E}_{j}, \ldots$ are built up of particular instances $\left(i_{1}, i_{2}, i_{3}, \ldots\right.$, and $\left.j_{1}, j_{2}, j_{3}, \ldots\right)$ that are equivalent in respect to a particular (intensional) aspect $(i, j, \ldots)$, such that these instances are mutually substitutable in the functional structure $f(\ldots, \ldots)$ with the image $A, B, \ldots$ The interpretation of this schema is easy for every case. In propositional $\operatorname{logic} \mathbf{E}_{i}$ and $\mathbf{E}_{j}$ represent the truth values $\mathrm{T}$ and $\mathrm{F}, i_{1}, i_{2}, i_{3}, \ldots$ all true and $j_{1}, j_{2}, j_{3}, \ldots$ all false sentences. $A$ and $B$ are the truth values of complex sentences (in this case, therefore, $A$ and $B$ coincide with $\mathbf{E}_{i}$ and $\mathbf{E}_{j}$ ), and the function $f$ (with arity 1 or 2 ) is one of the connectives $(\neg, \wedge, \vee, \rightarrow)$. The excluded or ignored intensional aspect in this case is the meaning (and even complexity) of each sentence: " $2+2=4$ " and "the sky is blue" are indistinguishable in propositional logic.

In semantics $\mathbf{E}_{i}, \mathbf{E}_{j}$, etc. represent different objects (references of singular terms) or properties (references of predicates), " $i_{1}, i_{2}, i_{3}, \ldots$ " different singular terms (or predicates) all of which refer to the same object ( $i$ ) (or property) and " $j_{1}, j_{2}, j_{3}, \ldots$ " different singular terms (predicates) which refer all to one other object $(j)$ (property). Note the interesting point: the intensional equivalence of the $i$-members (or of the $j$-members) is based here on co-referentiality, i.e. in having the same extension. The function $f$ is a $n$-placed predicate and $A$ and $B$ represent the truth-values $\mathrm{T}$ and $\mathrm{F}$. The excluded intensional aspect here is the meaning (Sinn) of the different singular terms (e.g meaning from "morningstar" and "eveningstar")-the truth value of the sentence is invariant to the mode of presentation. Exactly this intensional aspect becomes relevant in epistemic and modal contexts. Note that this kind of substitution is not only valid for coreferentiality: even if the predicates $F$ and $G$ express (intensionally) different properties (e.g. human and featherless biped), they can be substituted by each other when coextensional, too. The excluded semantical intensional aspect is the difference of (intensional) properties.

The interpretation for set theory is also easy. We can interpret $\mathbf{E}_{i}$ and $\mathbf{E}_{j}$ (etc.) as collections of elements and " $i_{1}, i_{2}, i_{3}, \ldots$ " as different ways of designating them (e.g. $i_{1}=\{11,13,17,19\}, i_{2}=\{19,17,13,12-1\}, i_{3}=\{x: x$ is prime and $10<x<20\}$, etc.). The functional structure $f(\ldots, \ldots)$ can be interpreted as any set theoretical relation (e.g. " $\subset$ ") and $A$ and $B$ as truth-values; thus, we can say that the true of set theoretical propositions is fully determined by the extension of a set, i.e. that sets with the same extension can always be substituted salva veritate. Or, alternatively, we can interpret $f(\ldots, \ldots)$ as an operation (e.g. " $\mathbf{E}_{i} \cup \mathbf{E}_{j}$ ") and $A$ and $B$ as the result of 
the operation; thus, we can say that set theory is extensional because all operations are fully determined by the extension of a set, i.e. the result of the operation is fully determined by the extension of the sets we operate. In any case, the excluded intensional aspect in set theory is the order, the way of designating and the nature of the elements.

Mereology is an extensional theory of collections for the same reason as set theory: its collections are invariant in respect to order or way of determining the elements. Thus, two mereological wholes A and B are fully determined by its parts, i.e. the whole is an extensional function of its parts. But we can say that mereology is even "more extensional" than set theory. In mereology another implicitly intensional element becomes neutralized, namely the hierarchy by the generation of the collection. Mereology represents an extensionalisation of the theory of collections in terms of the generation of complexes from the primitive elements. In set theory $\mathbf{E}_{i}$ represents the class of co-extensional collections $i_{1}, i_{2}, i_{3}, \ldots$ and the difference between $i_{1}$ and $i_{2}$ becomes irrelevant. Nevertheless, set theory still recognizes a difference between collections with the same primitive elements when these elements are collected at different levels, e.g. $\{m, n\} \neq\{\{m\},\{\{n\}\}\}$. The nesting of " $\{$ " and " $\}$ " in set theory generate new different entities (of new levels). In mereology this intensional distinction disappears, i.e. the hierarchical structure of levels is extensionalized and eliminated. For mereology $\{m, n\}$ is not only identical to $\{n, m\}$, but also to $\{m,\{n\}\}$, $\{\{m\},\{n\}\},\{m,\{\{n\}\}\}$, etc. If we take some primitive ur-elements to form a mereological collection, then the whole A (the mereological fusion) is fully determined by them, independently of order or level of generation. The generation of infinitely many sets in pure hierarchichal set theory has no correspondence in mereology.

The difference between set theory and mereology can also be shown by means of Marcus analysis. Take the principle:

$$
\text { If } \forall x\left(F x \mathrm{eq}^{*} G x\right) \text { then } F \mathrm{eq}^{* *} G \text {. }
$$

In set theory eq* must mean material biconditional and predication $F x$ is interpreted by means of $\in$. Thus, if all elements of $F$ are also elements of $G$ and vice-versa, $F$ is eq** (is identical with) $G$. But let us now interpret " $F x$ " mereologically as "participating in the constitution" or " $x$ is an element which occurs 'anywhere' in the collection $F$ "), then $F$ eq** (identical to) $G$ for mereology, but not necessarily for set theory.

The same kind of extensional reasoning could be applied to semiotics: $\mathbf{E}_{i}$ and $\mathbf{E}_{j}$ can be seen as equivalent classes of similar signs (e.g. $i_{1}=$ cat, $i_{2}=$ cat, $i_{3}=$ cat, ...) or sounds. We have to grasp different tokens of signs as instances of the same type. Without the ability of recognizing similar patterns, language (and also knowledge) it would become impossible. Another interpretation concerns meaning. If we interpret $\mathbf{E}_{i}$ and $\mathbf{E}_{j}$ as an equivalent class of synonymous signs or expressions ( $i_{1}$ 
$=$ "bachelor", $i_{2}=$ "not married", ...), that can be words or sentences, we have a classical conception of meaning (the meaning of a word or a proposition). Even enemies of the notion of meaning must assume such a kind of class of equivalence for explaining natural semantics: (relative) equivalent linguistic behaviour or (relative) equivalent stimuli. And since this class of equivalence is, strictly speaking, an intensional entity (as should be argued in the two next sections of this chapter), we could derive an argument for the objective existence of things like meanings and concepts (if someone defines concepts as meanings of words, contrary to this paper).

Let us extend the interpretation into the domain of propositional logic. Given the fact that for every sentence $\alpha$ and $\beta$, " $\alpha \rightarrow \beta$ " is a formal equivalent of " $\neg \beta \vee \alpha$ ", we could say that the complex sentences " $\alpha \rightarrow \beta$ " and " $\neg \beta \vee \alpha$ " (and also " $\neg(\beta \wedge \neg \alpha$ )", etc.) are substitutable salva forma logica. Thus, $\mathbf{E}_{i}(\{\alpha \rightarrow \beta\}$ or the logical form of conditional) is the class of equivalent formula $\left(i_{1}=\neg \beta \vee \alpha, i_{2}=\neg(\beta \wedge \neg \alpha)\right.$, etc.); $\mathbf{E}_{j}(\forall x(F x)$ or the logical form of general quantification) the class of the equivalent formula $\left(i_{1}=\neg \exists x \neg(F x)\right.$ ). I think this was Wittgenstein's insight in the Tractatus, which led him to the assertion that Russell's and Frege's logical notation were not fully adequate. In a correct notation, there could be no possibility of redundancy in logic. He suggested considering all equivalent forms like $\{\alpha \rightarrow \beta\},\{\neg \beta \vee \alpha\}$, $\{\neg(\beta \wedge \neg \alpha)\}$, etc. as reducible to the unique sequence $\langle\mathrm{T}, \mathrm{F}, \mathrm{T}, \mathrm{T}\rangle$.

To sum up, we can say in general that a context is extensional in respect to an aspect $P$ when it recognizes classes whose members are equivalent in respect to $P$, but different in respect to (intensional) property $Q$, such that they are substitutable preserving property $R$. Propositional logic is invariant in respect of the content of its propositions $(Q)$, just their truth-value $(P)$ are relevant for the purpose of logical operations $(R)$; extensional semantic contexts are invariant in respect to the mode of presentation of the reference $(Q)$, just the reference $(P)$ determines the truth value of sentences $(R)$; sets are invariant in respect to order or mode of presentation $(Q)$ of elements, just what elements compound a set $(P)$ is relevant for its identity and for set operations $(R)$; mereology is invariant in respect to level of composition (and order or mode of presentation of elements) $(Q)$, just what primitive elements compound the complex $(P)$ is relevant for the identity of fusions $(R)$.

A context is called intensional when we can recognize within this context fine differences overlooked in extensional contexts: the members of equivalent classes are recognized as different. We could also say that intensionality is characterized by its fine granularity, or by its higher degree of differentiation. These intensionally different entities form together a class of equivalence in which intensional differences "disappear" (they can be considered in this context irrelevant). But these differences are recognized in a more perspicuous analysis with the introduction of an "intensional" point of view. Insofar as predicate logic recognizes the difference of the content of true sentences (e.g. between " $2+2=4$ " and "Sky is blue"), it is, 
in some sense, more intensional than propositional logic. Insofar as propositional modal logic recognizes the differences between modi of being true (contingent or necessary truth), it is more intensional than non-modal propositional logic. Insofar as modal context and context of propositional attitude recognizes the difference between modes of presentation (e.g. "Plato" and "the author of Republic"), it is more intensional than direct contexts. Finally, insofar as set theory recognizes the difference between different levels of organization of collections of the same urelements; it is more intensional than mereology.

Now, how should we think about the relation between the two views of the intension-extension distinction? One could suppose that this second view is technically much more sophisticated and, thus, much more interesting than the first one. But I think that the reverse is the case. Note that the second view can be defined in terms of the first one. The set determined by a rule or intension (in the first view) forms an equivalent class, and the elements of this equivalent class are exactly the kind of substitutable instances of the principle of substitution that defines extensional contexts of the second view. Propositional logic is extensional because all instances (in this case, propositions) of the universal is true (and all instances of is false) are substitutable, predicate logic is extensional because all instances (in this case predicates) of coreferencial with a given predicate $F$ are substitutable, etc. In a word, every case of substitution salva veritate is defined by means of substitution of instances of a set (or equivalent class) of things that satisfy a given condition, i.e. that obey a certain rule. The particular instances $i_{1}, i_{2}, i_{3}, \ldots$ in the graphic above are instance of the rule $\mathbf{E}_{i}$. Substitutibility of extensional contexts is always grounded in the fact that different substitutable particular instances obey (are instances of) the same intensional rule. Thus, we could say that the first "metaphysical" view is more basic insofar as the second view depends on and can be defined in terms of the first one. And when I propose now that logic is essentially intensional, this should be understood according to the first view. Of course, if logic is a rule-governed activity and rules are intensional entities, logic is intensional in a trivial sense. But I think that logic is intensional in a stronger and more interesting sense.

\section{On the Intensionality of Logic}

For our analysis on the nature of logic we just need to remember: Intensions are "rule-type" entities, and extensions are collections of particular instances corresponding (or not corresponding, that's the matter in question!) to those rules. In order to show that logic is essentially intensional in my sense, I will argue that its central notion - that of logical consequence or deducibility-is intensional.

Now, before we begin with the argument concerning logical consequence, let us

Principia 14(10): 111-24 (2010). 
see an analogous case: the notion of identity. There are many questions concerning definition, criteria, etc. that philosophers have been trying to explain for centuries. Given any set $\{a, b, c, \ldots\}$ one could define identity as the diagonal of the Cartesian product of this set by itself. Of course, this definition only makes sense when we suppose a standard diagrammatic organization of sets:

$$
\begin{gathered}
\{a, a\},\{a, b\},\{a, c\},\{a, d\}, \ldots \\
\searrow \\
\{b, a\},\{b, b\},\{b, c\},\{b, d\}, \ldots \\
\searrow c, a\},\{c, b\},\{c, c\},\{c, d\}, \ldots \\
\searrow d, a\},\{d, b\},\{d, c\},\{d, d\}, \ldots
\end{gathered}
$$

Quite right! The diagonal set $\{\{a, a\},\{b, b\},\{c, c\},\{d, d\}, \ldots\}$ is indeed exactly the identity set. This is extensionally correct. But again: extensions do not determine intensions. It would be naive to think that with this definition we can solve any interesting philosophical question concerning identity. Note that "to be an element of the diagonal of the Cartesian product" is an intension in our sense: it is a rule, and so this definition is, in some sense, intensional. But this is a "wrong" intension. The notions of diagonal and of Cartesian product have no relevance for explaining in an interesting sense the concept of identity. There is no philosophical gain by analysing the notion of identity by means of the diagonal of the Cartesian product of a set. To be in the diagonal is only relevant for identifying univocally the identity set in virtue of a philosophically irrelevant diagrammatic strategy of organizing the sets of the Cartesian product of a given set. Now, let us see the logical notion of deducibility.

Logic needs a language to be formulated. Logicians usually take a set of primitive signs and syntactical rules which define well-formedness, i.e. the set of well-formed formulae (wff) of a language $L$, since not every string of primitive signs is an expression of the language. Syntactical rules are necessary for deciding which strings are genuine expressions of that language. The syntactical rules determine the extensional series of all (in general infinite) wff's. E.g. the rule "if $A$ is a wff of $L$ and $B$ is a wff of $L$, then $(A \wedge B)$ is a wff" "comprises" the collection " $a \wedge b, c \wedge(a \wedge b)$, $d \wedge(c \wedge(a \wedge b)), \ldots$. Even if we could start with the set of all possible strings of primitive signs of predicate logic (also including strings like " $x F \neg \rightarrow \forall F \exists$ ") as "already given in a kind of Platonic heaven", and if the syntactical rules were regarded as rules for "picking up" wff's from this set, these rules are intensional, in the same sense that the intension of the concept red "tells" us how to "pick up" red things in the world. It is not difficult to give an inventive rule for organizing formulae in a diagram such that wff's were in the diagonal of all strings of the primitive signs.

Principia 14(10): 111-24 (2010). 
Thus, one could say that because of intensionality of language, logic is also intrinsically intensional. But this would be overhasty. We could also extensionally define a language listing all wff $s$, in particular when we are modest enough to accept a language with finitely many expressions.

Now, a language becomes a logical system when we introduce rules for establishing valid inferences between sets of wff's. Thus, from the extensional point of view, we could say that the inference rules of a given system of logic define a subset of the Cartesian product $P(L) \times P(L)$ of wff's of $L$. But which subset? The diagonal one? Of course not. And exactly at this point logic become essentially intensional.

Logical systems have the power, given a finite set of rules, to regulate all logically valid inferences, i.e. to determine the relations of deducibility which subsist between subsets of wff's of $L$. In standard first order logic we distinguish three infinite sets of formulae: contradictions, tautologies and contingent formulae. The list of all (infinite) tautologies of a formal system of logic provides an extensional definition of this system. Of course, instead of giving the exhaustive list of all tautologies, we usually define a system listing the rules of inference. The rules of inference determines also for each contingent formulae $\alpha$ we take as premise three classes of formulae: all consequences of $\alpha$, all formulae incompatible with $\alpha$ and all formulae logically independent of $\alpha$. The interesting point here is the relation between the set of logical consequences of $\alpha$ and $\alpha$. We can define the general task of logic as establishing rules that define the relation of deducibility $\Gamma \vdash \Sigma$ : given any arbitrary set of premisses $\Gamma$ the rules determine the set $\Sigma$ of valid inferences. Given our general intension-extension schema

$$
R \quad \leftrightarrows \quad i_{1}, i_{2}, i_{3}, \ldots
$$

the $R$ represents in the case of logic the relation of deducibility and the instances $i_{1}$, $i_{2}, i_{3}, \ldots$ are ordered pairs of sets of wff's. Thus, for standard propositional logic e.g. $R$ is the intensional rule $\vdash_{\mathrm{PL}}$, and

$$
\begin{aligned}
& \langle\{\alpha\},\{\alpha, \alpha \vee \beta, \beta \rightarrow \alpha, \neg \neg \alpha, \ldots\}\rangle \\
& \langle\{\alpha \wedge \beta\},\{\alpha, \beta, \alpha \vee \beta, \beta \rightarrow \alpha, \alpha \rightarrow \beta, \ldots\}\rangle
\end{aligned}
$$

are the particular instances. Strictly speaking, each particular inference rule is an intension that determines a corresponding extension. Thus, modus ponens e.g. is a rule that determines the extension

$$
\begin{aligned}
& \langle\{\alpha, \alpha \rightarrow \beta\},\{\beta\}\rangle \\
& \langle\{\alpha \wedge \beta, \alpha \wedge \beta \rightarrow \gamma\},\{\gamma\}\rangle
\end{aligned}
$$

Principia 14(10): 111-24 (2010). 
Since a proof normally contains different rules combined in sequence, the set of instances $i_{1}, i_{2}, i_{3}, \ldots$ corresponding to the $\vdash$ is not simply the union of the sets of instances of each particular rule of the set. The ordered pair $\langle\{\alpha, \alpha \rightarrow \beta\},\{\beta\}\rangle$ e.g. is an instance of $\vdash_{\mathrm{PL}}$ although it is neither an instance of the rule modus ponens nor of the rule of elimination of conjunction.

The analogy to the intension-extension distinction made in the first section concerning concepts is easy: as the intension of a concept determines the "right" collection of all particular instances which fall under it, thus a logic determines "the correct" (or "one correct" for each different logic) set of wff's $(\Sigma)$ that follow from each set of premises $(\Gamma)$. My main point is: a pure extensional identification of all sets of formulae does not define the essence of a particular logic. This is Dummett's point, too, when he writes:

We can, thus, imagine a child being taught to discriminate, by syntactic tests, between valid and invalid arguments of some restricted kind (sentential arguments or syllogisms); if he is taught in a very unimaginative way, he may see the classification of arguments into valid and invalid ones as resembling the classification of poems into sonnets and non-sonnets, and so fail to grasp that the fact that an argument is valid provides any ground for accepting the conclusion if one accepts the premisses. (Dummett 1973: 454)

Even if we achieved "the" correct subset of the Cartesian product $P(L) \times P(L)$ we could not be sure that we understand the (intensional) concept of "classical logical consequence". Someone can simply recite the following set of ordered pairs:

$$
\begin{aligned}
& \langle\{\alpha\},\{\alpha, \alpha \vee \beta, \beta \rightarrow \alpha, \neg \neg \alpha, \ldots\}\rangle \\
& \langle\{\alpha \wedge \beta\},\{\alpha, \beta, \alpha \vee \beta, \beta \rightarrow \alpha, \alpha \rightarrow \beta, \ldots\}\rangle \\
& \ldots
\end{aligned}
$$

without grasping the intension of $\vdash_{\mathrm{PL}}$.

There are two questions we must distinguish here. First: given a particular logic (a particular set of ordered pairs of wff's), there is a subdetermination of its rules (there can be different sets of inference rules that determine the same set of ordered pairs of wff's). I am not claiming that different sets of rules that determine exactly the same set of ordered pairs of wff's are different logics: of course, different formulations of standard propositional logic are different formulations of the same logic. The extension determines the identity of a logic. In this sense, particular logical systems could be considered extensional: the criteria for identifying a system of logic are extensional. But this does not imply that logic is extensional in a more general sense. To individuate an entity is not the same as to give its essence or nature. My point could be stated in a crude manner: nobody that formulates a logical system examines the set of ordered pair of wff's (the list $i_{1}, i_{2}, i_{3}, \ldots$ ) and then 
searches for corresponding inference rules that generate this list. And the reason is a not a pragmatic or purely methodological one, but reflects the nature of making logic. And this is a first symptom for what I call here "the intensional nature" of logic.

Second, and more important for our question on the nature of logic: Why are we not willing to accept every set of ordered pairs of wff's as a particular "new" logic? And are there any criteria for deciding if a given set is a particular "new" logic or not? In this second question we reinterpret our graphic: given the concept of logic (it is the $R$ now), what particular sets of ordered pairs of wff's belong to the corresponding extension $i_{1}, i_{2}, i_{3}, \ldots$ ? Some formal properties are usually presented for defining the classical notion of logical consequence. The most common are:

(1) Consistency: the set $\Sigma$ of consequences must be consistent if the set or premisses $\Gamma$ is consistent,

(2) Reflexivity: if $\alpha \in \Sigma$, then $\Sigma \vdash \alpha$,

(3) Cut or transitivity: if $\Gamma \vdash \alpha$ and $\Gamma, \alpha \vdash \beta$ then $\Gamma \vdash \beta$,

(4) Monotonicity: if $\Gamma \vdash \alpha$, then $\Gamma, \beta \vdash \alpha$,

(5) Ex falso: $\alpha \wedge \neg \alpha \vdash \beta$.

But are we willing to accept that these properties really define the essence of logical reasoning? Most logicians deny this today. On the one side, there are many systems of logic that do not satisfy these properties (e.g. paraconsistent logic of da Costa) and today most of us are tolerant enough to accept them as "logical" systems. On the other side, as I will briefly show, we can construct a set of pairs that satisfy nearly all these formal properties, and we certainly would not accept it as a kind of logic. Should a logic satisfy some of them, maybe at least two of them? Which conditions? I think there is no rule for defining logic in general. How should we decide e.g. if $\alpha$ is an element of the set of conclusions of $\neg \neg \alpha$ or not? Logicians often argued in this manner: they show that some "intuitively valid arguments" are not expressible in standard logic, and that, because of this, we need a new logic with some deviant rules. (But, again, nobody examines a set of ordered pairs of wff's and tries to discover the corresponding rules for it.)

Let us suppose that we have the language $L$ and we define our collection of rules of inference $R^{*}$ such that, for every set of premisses $\Gamma$ there is a corresponding set of theorems $\Sigma$, such that $\Gamma=\Sigma$. This "logic" would be purely a game of reflexivity: from each formula $\varphi$ we could derive $\varphi$ and nothing more. $R^{*}$ satisfies the properties of consistency, reflexivity, transitivity and monotonicity. Only the rule of ex falso will not be valid, but not because of trivialization (from $\alpha \wedge \neg \alpha$ we could not infer $\beta$, but only $\alpha \wedge \neg \alpha$ ). Would we really say that this logic constitutes a good system of inferences? Some might respond positively, some negatively. The most evident 
failure of this "logic" is the absolute absence of any "new" derivable wff's. We miss the projective nature of logic-and this projective nature is intensional in my sense.

Another example: let us consider $L$ something like natural English and define the inference rule set $R^{* *}$ such that we get for every set of premises $\Gamma$, when neither "God exists" nor "God does not exist" are an element of $\Gamma$, the set of usual classical consequences $\Sigma$ plus "God exists". Thus, in this logic we have something like $\{\alpha \wedge \beta\} \vdash\{\alpha, \beta, \alpha \rightarrow \beta, \alpha \wedge \beta$, God exists, $\alpha \vee \beta, \ldots\}$. Would we accept this as a "genuine" logical system? I don't think so. Even satisfying most of the standard formal properties, we do not automatically get a logic. These two examples show that the formal properties are not sufficient for defining the essence of logic.

Now, let us see an example that shows that these formal properties are not necessary for defining the essence of logic. This is a short story with a metaphysical flavour. God is planning the creation of the world and in order to select the best one, He must first know the infinite set of all possible worlds. But which worlds are possible, how can He calculate what alternatives are available? In order to calculate this (of course, for classical metaphysics God does not need calculation, because He has immediate intuitive knowledge of everything, but this is not relevant here), He proceeds in the following manner: He takes (randomically or systematically, I do not know) an atomic proposition $\phi_{0}$ as starting point (this is His premise). Then He uses a rule we can call "rule of compossibility" $R^{C}$, i.e. He "infers" the set of propositions which are compossible with this proposition (e.g. $\left\{\phi_{1}, \phi_{2}, \phi_{3}, \ldots\right\}$, thus generating a set of pairs of facts in which $\phi_{1}$ is one element (e.g. $W_{1}=\left\{\phi_{0}, \phi_{1}\right\}$, $\left.W_{2}=\left\{\phi_{0}, \phi_{2}\right\}, \ldots\right) . W_{1}, W_{2}$, etc. are a kind of "open set", or "set in creation", like bottles into which God puts compossible facts. These are His new sets of "premises". Then, God applies the rule of compossible again, generating sets with three propositions as elements and so on, until He completes all maximal (let us suppose: finite) sets of compossible propositions-Lindenbaum told us that God can do this. I assume here that compossibility is not only a logical notion (in the sense that $\phi$ and $\neg \phi$ are not compossible), but also a material one (in the sense that $a$ is red and $a$ has no extension or a is not coloured are not compossible).

Is GodŠs reasoning a kind of "logic"? I think it certainly is. But note that the "rule of compossible" $R^{C}$ (the only rule used here) is not reflexive (if $\phi_{1} \in W$ (W is a determinate set at a given state), then it is not the case that $W \vdash_{R^{C}} \phi_{1}$, i.e. God does not introduce into a world $W$ a proposition that is already contained in $\mathrm{W}-\mathrm{He}$ does not waste time making a loop). $R^{C}$ is also non-monotonic (it is even "antimonotonic": the more propositions he puts into a world, the less are the compossible propositions He still can put into it). Further, the ex falso rule is not valid, or better, it is pointless because it will never come to the situation that $\phi \wedge \neg \phi$. The only principle GodŠs reasoning follows is transitivity: When God can put $\phi$ into the world $W$, and also $\phi^{*}$ into the world $W \cup\{\phi\}$, then He also can put $\phi^{*}$ into $W$. But there is a

Principia 14(10): 111-24 (2010). 
sense in which compossibility is not transitive (neither has a fixed arity). Take the propositions

$$
\begin{aligned}
& \mathrm{P}_{1}: a \text { is taller than } b, \\
& \mathrm{P}_{2}: \quad b \text { is taller than } c, \\
& \mathrm{P}_{3}: \quad b \text { is taller than } a, \\
& \mathrm{P}_{4}: \quad c \text { is taller than } a .
\end{aligned}
$$

$\mathrm{P}_{1}$ is compossible with $\mathrm{P}_{2}$ and $\mathrm{P}_{2}$ is compossible with $\mathrm{P}_{3}$, but $\mathrm{P}_{1}$ is not compossible with $\mathrm{P}_{3}$. Further, $\mathrm{P}_{1}$ is compossible with $\mathrm{P}_{2}, \mathrm{P}_{2}$ is compossible with $\mathrm{P}_{3}, \mathrm{P}_{3}$ is compossible with $\mathrm{P}_{4}$, but the four propositions are together not compossible.

Thus, the set of formal properties normally used to characterize which kind of sets of ordered pairs of wff's are logic and which aren't are neither sufficient nor necessary. We have some intuition about logicality not captured by them. There are many difficult metaphysical questions concerning identity, and nobody can suppose that with the extensional definition of identity (such as the diagonal of the Cartesian product of every set with itself) we can solve any such questions. This would be extremely naïve. The extensional definition of identity is no help in grasping the essence of identity. Similarly, the extensional characterization of logical deducibility by means of a function from subsets of sentences of $L$ to subsets of sentences of $L$ is inadequate toăreveal the philosophically interesting notion of logical deducibility. Logic as a science of inferences must be characterized in a more intensional way. Rules of inference are functions from sets of wff's to sets of wff's, but this relation cannot be an arbitrary relation, it must be an intensional function. What this "intension" should codify, this is one of the difficult questions of the philosophy of logic. ${ }^{1}$

\section{References}

Belnap, N. 1962. Tonk, Plonk, and Plink. Analysis 22: 130-4.

Dummett, M. 1973. Frege: Philosophy of Language. Cambridge, Mass.: Harvard University Press.

Marcus, R. B. 1971. Extensionality. In L. Linsky (ed.) Reference and Modality. Oxford: Oxford University Press.

Prior, A. N. 1960. The Runabout Inference-Ticket. Analysis 21: 38-9.

GUido IMAGUiRe

Universidade Federal do Rio de Janeiro (UFRJ)

Largo de São Francisco de Paula, n.1, 2 Andar

Rio de Janeiro

BRAZIL

guido_imaguire@yahoo.com

Principia 14(10): 111-24 (2010). 
Resumo. As expressões "extensão" e "intensão" são usadas de diferentes modos. Eu especifico na primeira parte deste artigo dois sentidos básicos da distinção e procuro mostrar que o antigo sentido metafísico, em termos de instância vs. universal, é mais fundamental que o sentido contemporâneo em termos de substitutividade. Na segunda parte eu argument que toda lógica, de modo geral, é essencialmente intensional, não apenas porque lógica é uma atividade guiada por regras, mas porque até a definição extensional de um sistema lógico pressupõe uma noção intensional de conseqüência lógica.

Palavras-chave: Extensão, intensão, conseqüência lógica.

\section{Notes}

${ }^{1}$ Thanks to Prof. Matthias Schirn for important discussions concerning Wittgenstein's Philosophy of Mathematics and to CAPES/DAAD program for supporting this investigation.

Principia 14(10): 111-24 (2010). 